\title{
Challenges and Opportunities to Advancing Telehealth: US Telehealth Resource Centers' Approach
}

\author{
Christina HIGA ${ }^{\mathrm{a}, 1}$ Elizabeth A. KRUPINSKI ${ }^{\mathrm{b}}$ \\ Deborah BIRKMRE-PETERS ${ }^{\mathrm{a}}$ and Sairel LABASAN ${ }^{\mathrm{a}}$ \\ ${ }^{a}$ Pacific Basin Telehealth Resource Center \& University of Hawai 'i at Mānoa \\ ${ }^{b}$ Southwest Telehealth Resource Center \& Emory University
}

\begin{abstract}
For the past thirty years, the United States Office for the Advancement of Telehealth has promoted the use of technology for health care, education, and health information services, and funds the National and Regional Telehealth Resource Centers (TRCs) to provide technical assistance to support stakeholder telehealth adoption. To assess the challenges and opportunities for the TRCs to advance telehealth, we reviewed publications, national and regional telehealth strategies, guidance from government agency reports and the TRC websites. We summarized information about the mission, funding and structure of the TRC program in terms of the shared service center model of organizational functioning, followed by a description of the TRCs' recent response to the COVID-19 Public Health Emergency.
\end{abstract}

\section{Introduction}

The Office for the Advancement of Telehealth (OAT) was established by the Health and Human Services Administration (HRSA) of the United States Department of Health and Human Services (DHHS) in the mid-1990s [1]. For the past thirty years, OAT's mission has been to promote the use of technology for health care, education and health information services. OAT funds various programs for telehealth network development, research, workforce development and technical assistance [2]. OAT funds the National and Regional Telehealth Resource Centers (TRCs) to provide technical assistance to support stakeholder telehealth adoption in the US [3].

There has been a steady increase in the adoption of telehealth in the US over the years with OAT and other government investments for technical assistance, but also infrastructure, equipment and telehealth program development [4]. For example, the Universal Service Program, administered by the Federal Communications Commission, allocates up to $\$ 400$ million annually for telecommunication and broadband infrastructure for rural health care providers [5]. The Rural Development section of the US Department of Agriculture supports a distance learning and telehealth program that typically funds end-user equipment at approximately $\$ 50,000-\$ 1,000,000$ per award [6].

${ }^{1}$ Corresponding author: Christina Higa, PhD, Pacific Basin Telehealth Resource Center, University of Hawai' ${ }^{i}$ at Mānoa - College of Social Sciences/ Social Science Research Institute, 2424 Maile Way, Saunders Hall 713 Honolulu, Hawai'i 96822; Email: christina@uhtasi.org 
Although there have been decades of work in advancing telehealth in the US, there are still varying levels of adoption across the country. Rapid and widespread telehealth adoption, however, occurred in response to the Coronavirus Disease 2019 (COVID-19) Public Health Emergency (PHE) [7,8]. The US government promoted telehealth as a means to meet PHE stay-at-home and social distancing mandates. Health care providers across the country needed to rapidly implement telehealth services, but were often unprepared and lacked the knowledge of what was required. The Telehealth Resource Centers (TRCs) were ideally positioned to help, as they were well established with ten to fourteen years of operational experience in providing telehealth technical assistance at national and regional levels. Thus, the TRCs' organizational structure and experience provided immediate resources for telehealth stakeholders in the US.

This paper will examine the Telehealth Resource Centers' challenges and opportunities for advancing telehealth in terms of organizational structure, range of services and other flexibilities to meet intense and rapidly changing needs for telehealth during the COVID-19 PHE.

\section{Methods}

To assess the challenges and opportunities for the TRCs to advance telehealth, we reviewed publications, national and regional telehealth strategies, guidance from government agency reports and the TRC websites. PubMed, Web of Science and Google Scholar were searched using the terms "telehealth resource center" and "TRC" for any relevant publications. Literature that focused on the Telehealth Resource Centers was included in our review while literature on other topics was excluded. We summarized information about the mission, funding and structure of the TRC program. The National Consortium of Telehealth Resource Centers' (NCTRC) organizational structure is discussed in terms of the shared service center model of organizational functioning. This is followed by a description of the TRC's recent response to the COVID-19 PHE and an overview of the challenges and barriers faced by the TRCs.

\section{Findings}

The search on PubMed, Web of Science and Google Scholar yielded very few relevant publications that described the Telehealth Resource Centers. The search term "telehealth resource center" yielded 1,753 results on PubMed, 196 results on Web of Science and 39,800 results on Google Scholar. A review of publication titles and abstracts revealed that the majority of the publications on the three databases were unrelated to the Telehealth Resource Centers. The results from Google Scholar were most promising as the search included some publications from the TRCs themselves. Given the very few publications that met the inclusion criteria, our findings were acquired from national and regional telehealth strategies, reports from government agencies, and the information available on the TRC websites.

\subsection{Statutory Authority and Appropriations of the Telehealth Resource Centers}

The Telehealth Resource Center program was established by law under the healthcare safety net amendment of 2002 (P.L.107-251, as amended) that was amended in 2020 by 
the Coronavirus Aid, Relief, Economic Security Act (CARES Act; P.L. 116-136). By statute, the TRCs are designated to provide technical assistance, generally at no cost, to telehealth stakeholders, including state and local health care facilities, health care administrators, chief financial officers, health care providers and patients $[2,9,10]$. The main purpose of the TRC Program is to provide expert and customized telehealth technical assistance throughout the US. The TRCs are located regionally to facilitate activities at the local level and to ensure that resources are geographically distributed. This regional aspect also helps ensure cultural understanding and appreciation of the unique populations and issues within each region. The TRCs provide training and support, disseminate information and research findings, promote effective collaboration, and foster the use of telehealth technologies to provide health care information and education for health care providers who serve rural, frontier, and medically underserved areas and populations. The TRCs share expertise through consultations, training, webinars, conference presentations, and a significant web presence.

The Office for the Advancement of Telehealth (OAT), Health and Human Services Administration (HRSA) of the United States Department of Health and Human Services (DHHS) has administered the grant program since 2002, with funds being made available in FY2005. The first cycle of three-year grants started in FY2006 [11]. HRSA telehealth appropriations in FY2020 was \$28.5 USD million. This includes the TRCs' funding that has ranged from $\$ 300,000$ to $\$ 325,000$ USD annually per resource center [12].

\subsection{TRC Organizational Structure}

Initially the TRCs were funded through a grant mechanism, but in recent years cooperative agreements have been used. A cooperative agreement is a financial assistance mechanism where HRSA expects substantial involvement with the recipient during project performance. Whereby HRSA can among other things, identify special projects or studies for the TRCs to conduct, participate in planning of strategic direction of services provided by the TRCs and provide input and background on current and future issues.

There are twelve Regional TRCs, with three being funded in 2006 - California Telehealth Resource Center (CTRC), Great Plains Telehealth Resource \& Assistance Center (gpTRAC) and Northwest Regional Telehealth Resource Center (NRTRC). In 2009 the Southwest Telehealth Resource Center (SWTRC), Pacific Basin Telehealth Resource Center (PBTRC), South Central Telehealth Resource Center (SCTRC) and Southeastern Telehealth Resource Center (SETRC) were established and funded. In 2010 the remaining TRCs were funded: Heartland Telehealth Resource Center (HTRC), Upper Midwest Telehealth Resource Center (UMTRC), Mid-Atlantic Telehealth Resource Center (MATRC), TexLa Telehealth Resource Center (TexLa) and Northeast Telehealth Resource Center (NETRC). There are two National TRCs: the Telehealth Technology Assessment Resource Center (TTAC) was established in the 2006 funding cycle and the Center for Connected Health Policy (CCHP) in the 2010 funding cycle. An interactive map developed by the TRCs shows the regions covered by each TRC (https://www.telehealthresourcecenter.org/who-your-trc/).

Recognizing that resources needed to be used more efficiently and duplication reduced, in 2017 the TRCs agreed to work collaboratively as the National Consortium of Telehealth Resource Centers (NCTRC). The evolution of the NCTRC is analogous to the concept of 'shared service centers' $[13,14]$. The concept of shared service center strategy generally has several characteristics to maximize operational efficiency, 
improve consistency and reliability across different units. Shared service center strategies were typically focused on transactional and routine processes in finance, administration (such as procurement, Human Resources) and IT (Information Technology), however, the main concepts of the theory align with the evolution of the NCTRC. The following features are generally accepted as characterizing SCCs [14].

- They result as a function of consolidation of processes to avoid duplication of work and to achieve synergies.

- Are exclusively engaged in support services to facilitate core processes of the entity, but are not core processes themselves.

- A main goal is reduction of costs by making use of economies of scale.

- They have a service-oriented focus on clients to optimize the client experience by focusing on service output.

- They align with external competitors to enhance competitiveness by building strategic knowledge, analyzing their own strengths and weaknesses thus illustrating their competitiveness to clients.

- Each unit is an independent organization clearly separate from others, with its own responsibilities and management.

- They are operated like a normal business managed like third-party vendors that tailor their services to the requirements of their customers.

As a Consortium, the TRCs have accumulated an unparalleled amount of resources to advance telehealth programs regionally and nationally. A charter was created and agreed upon by all TRCs. Important governmental aspects include, among others, the following:

- $\quad$ Each TRC is allowed only one vote on NCTRC issues and decisions are made by majority vote.

- NCTRC holds regular meetings: calls with HRSA and committee meetings, i.e., Metrics Committee, Governance Committee, and Strategic Planning Meeting Committees.

- Small committees can be formed to focus on specific work.

- TRCs agree upon an amount paid annually to the NCTRC and pay invoices in a timely manner.

In terms of administration, the TRCs collectively select one TRC to act as the Consortium Administrator that is responsible for all NCTRC operational duties. The Administrator will act as a main point of contact for communications. The Consortium Administrator is responsible for employing and supervising the NCTRC Project Coordinator (supported by the annual fees noted above). The Administrator processes and houses all contracts for the NCTRC (e.g., NCTRC booths at national conferences). The Administrator administers the NCTRC budget as approved by the Governance Committee and at the end of each grant year, prepares a proposed budget for the Governance Committee to discuss, finalize and approve.

The TRCs are designed for effective provision of technical assistance to health providers so they can efficiently integrate telehealth in their practices. Other studies have reviewed the potential for "shared service centers" for telehealth. For example, Denmark's SSC project found it important for the health care system to focus on internal operations such as "clinical effectiveness of an intervention, or cost effectiveness of a specific workflow." However, they found that the enabling role of a shared service center 
"may be characterized as external aspects, seen in relation to the individual telemedical initiative, as they create value on more general levels, such as scalability of services, cross-sector cooperation, and continuity" [13].

\subsection{Type of Technical Assistance}

The TRCs do not provide direct clinical care, but rather their mission is to:

a. Provide technical assistance, training and support for health care providers and stakeholders

b. Disseminate information and research findings related to telehealth services

c. Promote effective collaboration among TRCs and the Office for the Advancement of Telehealth (OAT) within HRSA.

d. Conduct evaluations to determine the best use of telehealth technologies to meet health care needs

e. Promote the integration of the technologies used in clinical information systems with other telehealth technologies

f. Foster the use of telehealth technologies to provide education for health care providers and consumers in a more effective manner; and

g. Implement special projects or studies under the direction of OAT [2]

In order to accomplish these goals, each of the TRCs is composed of experts from a variety of backgrounds who have been involved extensively in telehealth in a variety of ways (e.g., clinical practice, administration, research, program development), in some cases for well over 30 years. Individually, the TRCs have their websites highlighting their specific areas of expertise and the NCTRC has a central website housing key resources and tools for telehealth program development. At both the regional TRC and national NCTRC levels these experts provide webinars featuring key topic areas presented by TRC members as well as experts in telehealth from around the country and even the world. There are repositories of previously broadcasted webinars that can be freely accessed for future viewing and calendars of upcoming events.

TRC members regularly present at national and international conferences, host information booths at conferences, health fairs and a variety of other venues where healthcare providers, organizations and patients attend. A number of them also publish regularly in research journals and all disseminate information about telehealth via other methods such as newsletters, blogs and social media. They generate white papers, position statements, tools, templates and toolkits that help provide unified and standard language defining various aspects of telehealth. Collectively, they represent a "brain trust" of resources, information, experience and expertise that is unparalleled in the US.

The TRCs provide technical assistance to all types of healthcare organizations and providers. They provide assistance on a wide variety of topics, but some of the more common topics include:

- Needs assessments

- Identifying funding opportunities \& helping with grant preparation

- Designing \& implementing clinical \& administrative protocols

- Assisting with selection of technologies

- Assisting with telehealth licensure, credentialing and reimbursement laws

- Evaluation of program implementation \& sustainability efforts

- Market analyses, strategic and business planning 
- Information on obtaining third party reimbursement for telehealth services

- How to secure Medicaid waivers for telehealth

- Helping overcome licensure \& credentialing barriers

- Incorporating telehealth in health system reform initiatives

- Leveraging health information technology (HIT) infrastructures

- Collecting data $\&$ helping craft language for educating \& informing legislatures on telehealth regulations \& policies

\subsection{Telehealth Response to the COVID-19 Pandemic}

The overwhelming surge and widespread scaling up of telehealth services happened in the US and globally as a response to COVID-19 public health measures to reduce disease exposure for patients, families and health care staff. The use of telehealth also helped to lessen reliance on personal protective equipment (PPE) when there was PPE inventory shortage concerns.

To facilitate the widespread adoption the US government instituted temporary PHE policy changes and regulatory waivers from the Centers for Medicare and Medicaid Services (CMS). In the US, health care coverage is primarily categorized as public (federal, state) and private (private and self-insured). During the PHE federal policy changes did not always align with state laws. Medicare is a federal program that provides health care coverage for the disabled and elderly. Medicare policy and regulatory changes improved restrictive pre-COVID-19 telehealth reimbursement, increased the eligible provider and service type for telehealth reimbursement, waived or reduced patient co-payments, and allowed patients to be seen from home (rural or urban) instead of in a previously required rural health care setting. The US states and territories have oversight of Medicaid, private and self-insured coverage. Medicaid provides health care coverage for low-income beneficiaries. Although the regulatory and policy changes were primarily geared towards Medicare, states often followed the changes for Medicaid and private payor insurers. Legislative and regulatory changes were made at unprecedented rates in terms of the number of changes and frequency. A major role of the TRCs was to help track these changes at a national and state level.

As part of the CARES Act funding each TRC received \$828,571 USD in FY2020 to meet the extraordinary increase in technical assistance demands [12]. The TRCs across the country worked together to scale-up technical assistance in response to the COVID19 related telehealth surge. Each TRC experienced a significant increase (often more than doubled) in technical assistance requests, especially with respect to basic issues, such as, what technology to use, how to start a program, what were the changes in policy, and perhaps most commonly, what codes should be used for billing. The TRCs also experienced a significant increase in the number of organizations requesting training webinars and ready-made materials (e.g., consent templates) for rapid implementation. The number of patients reaching out for information about telehealth also increased dramatically, resulting in an increase in webinars and the creation of COVID-19dedicated resource materials available on the NCTRC and individual TRC websites.

\section{Discussion}

The strengths of the TRCs have proven to be numerous, especially during the COVID19 PHE. They have organized technical assistance to all states, US affiliated Pacific 
Island territories and freely associated states via the Regional TRCs and have been especially useful with respect to helping clarify the fragmented nature of US policies at the state level and sometimes at the federal level. The true value of the regional TRCs is the fact that the US is incredibly diverse in terms of geography and unique populations (e.g., Native Peoples) within each region. Each TRC has in-depth knowledge about telehealth and healthcare in general in their regions and has established connections and collaborations with communities of all types within their regions. This regional knowledge builds trust in TRC services and aids in expanding their technical assistance networks and ultimately their impact on patient care as they understand and appreciate health system resources and infrastructure, culture, language, expectations and local and state regulations and policies. The national TRCs are trusted sources for vendor neutral technology review and evaluation and the go-to experts for tracking federal policies as well as providing regular updates about state policy with a semi-annual summary guide of the nation's most current Medicaid provider manuals, applicable state laws, and telehealth-related regulations for all fifty states and the District of Columbia.

The NCTRC adds a layer of collaboration that creates a nationwide network of information and contacts that makes it easy to share best practices across the country and serve as a mechanism for TRCs to reach out and provide resources and support to each other when additional information on a topic is required. The Consortium also allows for increased efficiency through joint webinar production, tool kit development, infographic creation and joint hosting and staffing of booths at conferences. All of these collaborative activities not only reduce redundancies and increase efficiencies, but serve as a costsaving mechanism as well. In addition, the collaboration fosters collection and curation of information across the country through joint surveys, best practices and related efforts to create a unified picture of telehealth use and progress at the national level.

Despite the efforts and strengths on the TRCs over the years, there are still some challenges that they face. Requests for services and the types of organizations who reach out have increased with each year of the TRC existence. This increase was especially substantial during the COVID-19 pandemic and will likely continue afterwards as practices adjust to expiration of the COVID-19 waivers. Additionally, there has been a significant new focus on direct to consumer, patients and families with associated information requests from these groups. Although all this increase in technical assistance requests is desirable, as it demonstrates that telehealth is growing, funding levels have remained stagnant since the inception of the TRC program (with the exception of CARES funds which are for only one year). Since the TRCs are federally funded, they are also required to submit competitive applications for each funding cycle, thus there are no guarantees that a given TRC will be funded. If a new organization receives a grant as opposed to an existing TRC there is also no requirement from HRSA that they join the NCTRC, although they are encouraged to do so. This lack of potential continuity and consistency could negatively impact the NCTRC structure and the quality of services provided within each region.

Other telehealth barriers include systemic challenges for telehealth adoption. For instance, digital inequity in terms of access to broadband, devices, and digital literacy for health can prevent telehealth adoption. The TRCs are involved in digital equity and digital literacy working groups and task forces, but these groups are often not exclusively focused on telehealth. COVID-19 highlighted major disparities and we need to be careful that increased access to telehealth does not widen these existing disparities. In terms of policy, the TRCs have experience and expertise in all aspects of telehealth and regularly 
serve as telehealth advocates. The TRCs are prohibited, however, from government advocacy since they are funded by federal grants.

In conclusion, the NCTRC and its member TRCs represent a unique model of telehealth assistance in the US and can perhaps be used as a model globally. Given the various backgrounds and expertise of the NCTRC members, they are poised to meet the challenges of the changing landscape of telemedicine in the future and the implementation of new and developing technologies to better impact patient care and the health of the US.

\section{References}

[1] Doarn CR, Pruitt S, Jacobs J, et al. Federal efforts to define and advance telehealth--a work in progress. Telemed J E Health. 2014;20(5):409-418. doi:10.1089/tmj.2013.0336

[2] Pruitt S. The Office for the Advancement of Telehealth. Telemed eHealth 2013;19(5):346-348.

[3] Kwong MW, Calouro C, Nasseri L, Guitierrez M. The federally funded Telehealth Resource Centers. Perspectives of the ASHA Special Interest Groups 2015;5(1):14-23.

[4] McKinsey \& Company. Telehealth: a quarter-trillion-dollar post-COVID reality? May 29, 2020. Available at: https://www.mckinsey.com/industries/healthcare-systems-and-services/ourinsights/telehealth-a-quarter-trillion-dollar-post-covid-19-reality\#

[5] Universal Service. Available at: https:/www.fcc.gov/general/universal-service. Accessed November $30,2020$.

[6] United States Department of Agriculture Rural Development Distance Learning \& Telemedicine Grants. Available at: https://www.rd.usda.gov/programs-services/distance-learning-telemedicinegrants. Accessed November 30, 2020.

[7] Centers for Disease Control \& Prevention. Trends in the use of telehealth during the emergence of COVID-19 pandemic - United States, January - March 2020. Available at: https://www.cdc.gov/mmwr/volumes/69/wr/mm6943a3.htm. Accessed November 30, 2020.

[8] US Department of Health \& Human Services. Office of the Assistant Secretary for Planning \& Evaluation Issue Brief: Medicare beneficiary use of telehealth visits: early data from the staret of the COVID-19 pandemic. Available at: https://aspe.hhs.gov/pdf-report/medicare-beneficiary-usetelehealth. Accessed November 30, 2020.

[9] Elliott VL. The National Consortium of Telehealth Resource Centers: COVID-19 Assistance. Natl Consort Telehealth Resour Centers COVID-19 Assist . 2020;1. Available at: https://www.telehealthresourcecenter.org/

[10] Blanchet K. Innovative programs in telemedicine: Great Plains Telehealth Resource and Assistance Center. Telemed eHealth 2008;14(9):870-874.

[11] The National Consortium of Telehealth Resource Centers. A Decade of Serice: A Report on Ten Years of Service - with an Eye to the Future. https://www.telehealthresourcecenter.org/wpcontent/uploads/2018/05/TRC-10-Year-Report-digital-FINAL.pdf Accessed December 3, 2020.

[12] Health Resources \& Services Administration Telehealth Programs. Available at: https://www.hrsa.gov/rural-health/telehealth Accessd December 3, 2020.

[13] Larsen SB, Sørensen NS, Petersen MG, Kjeldsen GF. Towards a shared service centre for telemedicine: Telemedicine in Denmark, and a possible way forward. Health Informatics J. 2016. doi:10.1177/1460458215592042

[14] Janssen M, Schulz V, Brenner W. Characteristics of shared service centers. Transform Gov People, Process Policy. 2010. doi:10.1108/17506161011065190 